\title{
Desigualdades Horizontales, Guerras y Paz Violenta en el Norte de Uganda
}

\section{Horizontal Inequalities, Wars and Violent Peace in Northern Uganda}

\author{
Mauricio Uribe López ${ }^{1}$ \\ Universidad EAFIT (Colombia)
}

Recibido: 27-02-17

Aprobado: 19-12-17

\section{Resumen}

Este artículo explica la relación entre desigualdades horizontales y dos guerras consecutivas en Uganda: aquella que condujo a Museveni al poder en 1986 y la guerra de veinte años que este libró contra el Ejército de Resistencia del Señor. La investigación presenta la definición de la enemistad política con base en la disputa norte-sur, entre nilóticos y bantúes, como el mecanismo explicativo que conecta las desigualdades horizontales tanto con la dinámica de la guerra civil, como con el carácter violento del postconflicto ugandés, en un escenario marcado por el autoritarismo y el clientelismo.

Palabras-clave: Uganda, Acholis, Ejército de Resistencia del Señor, desigualdades horizontales, paz violenta, clientelismo.

\begin{abstract}
This article explains the role played by horizontal inequalities in two wars fought in Uganda: First, the one which led Museveni to power in 1986 and in

\footnotetext{
${ }^{1}$ (muribe14@eafit.edu.co). Doctor en Ciencia Política de la Facultad Latinoamericana de Ciencias Sociales - Sede académica de México. Profesor Titular del Departamento de Gobierno y Ciencias Políticas de la Universidad EAFIT (Medellín, Colombia). Entre 2005 y 2014 fue profesor del Centro Interdisciplinario de Estudios sobre Desarrollo de la Universidad de los Andes (Bogotá, Colombia), entidad que financió la investigación en la que se basa este artículo. Investiga temas relacionados con estatalidad, conflictos armados y construcción de paz y con los debates sobre elección social y justicia distributiva. Entre sus principales publicaciones recientes se destacan: La nación vetada: estado, desarrollo y guerra civil en Colombia (2013), Civil wars and violent peace in Africa and Latin America: A general outlook (2013), Elección social, desigualdad y virtud cívica (2017) e Infraestructuras de paz y estatalidad en Colombia (2018).
} 
the war he waged against the Lord Resistance Army. The research lays out how the north-south cleavage between Nilotic and Bantu peoples can be identified as a major explicative mechanism which connects horizontal inequalities to the civil wars' dynamics and to the violent post-conflict. It also shows the way in which violent peace in Uganda occurs within a milieu of authoritarianism and cronyism.

Key-words: Uganda, Acholi people, Lord Resistance Army, Horizontal inequalities, Violent peace, Crony capitalism.

\section{Introducción: La guerra en el norte de Uganda}

¿Por qué puede afirmarse que Uganda está en postconflicto y al mismo tiempo recibir la calificación de "conflicto armado interno internacionalizado"? La guerra civil representada en la díada Gobierno - Ejército de Resistencia del Señor (Lord Resistance Army LRA) liderado por Joseph Kony, dejó de librarse en Uganda desde 2006 sin que el LRA fuera derrotado y sin haber firmado un acuerdo definitivo de paz. La guerra se exportó a otros países de la región: Sudán del Sur, la República Democrática del Congo RDC y la República Centroafricana. En palabras del Profesor Phillip Apuuli Kasaija del Departamento de Ciencia Política y Administración Pública de la Universidad de Makerere: "La guerra no ha terminado aún. No se ha firmado un acuerdo de paz y el LRA no ha sido derrotado. Así que, técnicamente, el fin de la guerra no ha llegado todavía".

Las ofensivas del gobierno del presidente Yoweri Museveni contra los rebeldes en el norte de Uganda, especialmente la Operación Norte de 1991, llevaron al LRA a establecer su retaguardia en el sur de Sudán. El LRA obtuvo apoyo logístico y financiamiento por parte del régimen de Jartum presidido por Omar Bashir (Allen y Vlassenroot 2010) el cual, en ese entonces, libraba la que se conoce como la segunda guerra civil sudanesa $(1983-2005)^{3}$ contra el Ejército de Liberación del Pueblo de Sudán (Sudan's People Liberation Army SPLA) liderado por John Garang, amigo y aliado del presidente Museveni. Así las cosas, la guerra ugandesa se internacionalizó con el LRA actuando, en lo que hoy es Sudán del Sur, como uno de los brazos armados del gobierno de Bashir. Además, el ejército ugandés (UPDF Uganda People Defense Force) llevó sus operaciones más allá de su frontera norte.

La difícil posición internacional del régimen de Jartum -señalado durante los años noventa como aliado del terrorismo por el gobierno del presidente

\footnotetext{
${ }^{2}$ Entrevista en Kampala, 30 de septiembre de 2014.

${ }^{3}$ La primera inició a mediados de los años sesenta y terminó con el acuerdo de Addis Abeba en 1972.
} 
Clinton-empeoró tras los ataques del 11 de septiembre de 2001 en Nueva York. Esto obligó a Omar Bashir a romper con el LRA -al menos públicamente- y a aceptar la incursión del UPDF en 2002 en territorio sudanés. Museveni puso en marcha en el norte de Uganda y en el sur de Sudán la Operación Puño de Hierro con la que prometía poner fin al LRA. El régimen de Jartum y el SPLA firmaron un acuerdo de paz en 2005, lo que llevó a la conformación del gobierno autónomo de Sudán del Sur cuya independencia se concretó en el referendo de 2011. Desde 2005, el gobierno de Sudán del Sur inició contactos con el LRA y con el gobierno de Uganda, los cuales llevaron al inicio formal de los diálogos de paz de Yuba en julio de 2006. En febrero de 2008, la firma de un acuerdo de paz entre el LRA y el gobierno de Museveni parecía inminente. Sin embargo, Kony - contra quien la Corte Penal Internacional CPI emitió orden de detención desde $2005^{4}$ - se rehusó varias veces a firmar y en noviembre de 2008 se abandonaron los diálogos de Yuba. Los gobiernos de Uganda, Sudan del Sur y la RDC, pusieron entonces en marcha la Operación Trueno Luminoso que buscaba eliminar al LRA.

Joseph Kony y sus comandantes más cercanos, que habían estado refugiados en el extremo norte de la RDC, trasladaron desde $2008 \mathrm{su}$ principal zona de operaciones hacia una franja que va desde las inmediaciones del Parque Nacional Garamba en la RDC, hasta el extremo oriental de la República Centroafricana. ${ }^{5}$ La guerra no ha terminado pero los distritos acholi en el norte de Uganda (Gulu, Kitgum, Pader y Amuru) llevan, desde que empezaron los -a la postre fallidos- diálogos de Yuba, casi una década de postconflicto. En 2010, Ronald Atkinson, profesor de estudios africanos de la Universidad de Carolina del Sur, describía la situación en los siguientes términos: "En el norte de Uganda, entretanto, a pesar de la incertidumbre causada por la naturaleza intermitente de los diálogos de paz de Yuba, una transición en el terreno desde la guerra hacia la paz estaba en curso" (Atkinson 2010a, 220).

Uganda es un ejemplo sobresaliente de lo que en los estudios sobre desarrollo se conoce como "desigualdades horizontales" (Stewart 2009; Cederman, Gledisch y Buhaug 2013). Como otros países sometidos al dominio colonial europeo, Uganda heredó rivalidades internas que han moldeado las estrategias políticas basadas en la manipulación de las identidades étnicas

\footnotetext{
${ }^{4}$ El 16 de diciembre de 2003 la Oficina del Fiscal de la CPI recibió una remisión de parte del gobierno de Uganda sobre el caso del LRA, solicitando la apertura de una investigación. El 27 de septiembre de 2005 fueron transmitidas a los gobiernos de Uganda, RDC y Sudán, órdenes de arresto en contra de cinco comandantes del LRA incluyendo a Joseph Kony y a su número dos, Vincent Otti. El 13 de octubre de ese mismo año, los jueces de la CPI publicaron las órdenes en su totalidad (Brubacher 2010).

5 Según Francis Arop Omuk, director ejecutivo de Community Rural Empowerment and Support Organisation-CRESO, la autonomía y posterior independencia de Sudán del Sur tuvo un impacto negativo determinante sobre el LRA y lo obligó a desplazarse hacia el occidente hasta la República Centroafricana. Entrevista llevada a cabo el 23 de septiembre de 2014 en la población de Gulu.
} 
y regionales. La política ugandesa ha acentuado a su vez esas divisiones y ha convertido la violencia personal y directa, en un recurso ordinario en la búsqueda y mantenimiento del poder político y económico por parte de cada grupo en el poder, desde su independencia en 1962.

El artículo explora en el caso de Uganda dos hipótesis de trabajo: aquella que relaciona desigualdades horizontales (en sus dimensiones políticas y económicas) con el inicio y prolongación de las guerras civiles en contextos de autoritarismo y debilidad estatal (Cederman, Gledisch \& Buhaug 2013), y la que atribuye, a esas desigualdades, un papel relevante en la configuración de escenarios de postconflicto violento y represivo (Uribe López 2013). El texto está dividido en seis secciones, incluyendo esta introducción. La segunda sección se ocupa de la relación entre desigualdades y violencia política. La tercera describe la forma en la que se configuró la distinción entre nilóticos y bantúes, como clave en la definición de la enemistad política y en las oportunidades de manipulación para el logro y mantenimiento del poder. La cuarta sección presenta algunos aspectos centrales de la política contrainsurgente en el norte de Uganda. La quinta sección señala el carácter represivo y violento del postconflicto ugandés. Finalmente, se presentan algunas conclusiones.

\section{Desigualdades horizontales y violencia política}

Las desigualdades horizontales son desigualdades entre grupos que comparten cierta identidad y que se dan en una o varias de las siguientes cuatro dimensiones: participación política, activos (tierra, capital, crédito, recursos naturales), oportunidades laborales e ingresos y, acceso a los servicios sociales (educación, servicios de salud, agua potable, vivienda). La identidad con base en la cual se diferencian los grupos puede estar definida por vínculos de clase, lazos religiosos o étnicos, afiliaciones raciales u otras características relevantes que mantienen unidos a conjuntos significativos de personas (Stewart 2009). También adscripciones territoriales o regionales específicas en la medida en que-aclara Stewart-las desigualdades horizontales pueden estar espacialmente distribuidas.

Las desigualdades verticales en cambio, corresponden a desigualdades en la distribución de los ingresos o de los activos entre individuos u hogares en el conjunto de la sociedad. Múltiples ejemplos muestran que la desigualdad en la distribución del ingreso no es condición suficiente de conflicto armado. Hay países muy desiguales cuya historia reciente no registra guerra civil. Igualmente, aunque no abundan los ejemplos para descartar la desigualdad (horizontal o vertical) como condición necesaria de guerra civil, es posible hallar casos en los que guerra e igualitarismo coinciden. En Camboya, por ejemplo, la política 
tipo lecho de Procusto puesta en marcha por Pol Pot entre 1975 y 1979, no evitó que la situación de conflicto armado se prolongara hasta 1998.

Un argumento similar aplica para la relación entre desigualdades horizontales y guerras civiles. Ciertamente, "el conflicto violento tiene muchas causas económicas y políticas -bajos ingresos, alto desempleo, competencia por recursos, violaciones de derechos humanos, etc.- algunas asociadas con las desigualdades horizontales y otras no" (Stewart 2009:136). Malasia es un ejemplo de desigualdad horizontal (asociada a la ventaja económica de la minoría china), que produjo algunos motines a comienzos de los años setenta, pero que no desembocó en guerra civil. Sin embargo, es imposible explicar conflictos armados como los de Guatemala, Mozambique, Irlanda del Norte, Sri Lanka, Bosnia-Herzegovina, Ruanda, Burundi, Etiopía, Turquía, Indonesia, Siria, Sudáfrica y por supuesto, Uganda, sin considerar el papel desempeñado en esos casos por diversas dimensiones de desigualdad horizontal, incluyendo -como ha sido evidente en Etiopía, Turquía e Indonesia- la presencia de reivindicaciones secesionistas.

Algunos países con severas desigualdades horizontales no tienen altos índices de desigualdad vertical. Por ejemplo, Burundi y Etiopía. Mozambique -que padeció una dura guerra entre 1977 y 1992- no tiene una elevada concentración de la tierra ${ }^{6}$, y sin embargo, es un ejemplo muy claro de desigualdad horizontal.

Descartar las desigualdades, tanto verticales como horizontales, como condición necesaria o suficiente de una guerra civil, no las excluye como un factor relevante en la explicación de un amplio conjunto de situaciones de guerra civil. Carles Boix (2008) sometió a prueba la hipótesis según la cual el riesgo de violencia política, aumenta en presencia de una alta desigualdad en la distribución de la tierra ${ }^{7}$ y una elevada especificidad de los activos ${ }^{8}$. Usando la base de datos del Correlates of War Project y la de James Fearon y David

${ }^{6}$ El Gini de tierras de Mozambique es de sólo 0,368 (Frankema 2009). Aunque la cifra corresponde a 1999, hay que tomar en cuenta que si el coeficiente de Gini del ingreso es muy estable en el tiempo, aún más estable es el de la tierra: "De hecho, un estudio de datos de panel para 49 países encontró que el $91,8 \%$ de la varianza en la desigualdad se debía a la varianza entre países y que solamente el $0,85 \%$ era atribuible a la varianza a lo largo del tiempo" (Kanbur y Squire 2002: 183-184).

${ }^{7}$ Boix (2008) plantea inicialmente su hipótesis en términos de desigualdad en la distribución del ingreso. Sin embargo, considera que en un contexto de elevada desigualdad en el ingreso, aumentaría la demanda por redistribución. Esto podría conducir a mayores tarifas tributarias. Los activos menos específicos podrían trasladarse a otros países en los que las expectativas de un aumento en los impuestos sería menor, de modo que la demanda redistributiva caería más directamente sobre los activos más específicos, por ejemplo, la tierra.

${ }^{8}$ El grado de especificidad de un activo corresponde a la diferencia entre los beneficios que éste genera al interior del país y los beneficios que ese mismo activo podría generar si se traslada al exterior. Activos de fácil liquidación serían muy poco específicos y por tanto, menos "gravables" en la medida en que el inversionista puede reaccionar más fácilmente -ante un aumento de los impuestos- llevándose su inversión a otro lado. "Por ejemplo, algunos activos, tales como la tierra o los pozos petroleros, son completamente específicos al país en el que están localizados, no pueden ser trasladados a algún otro lugar" (Boix 2008: 201).

Araucaria. Revista Iberoamericana de Filosofí, Politica y Humanidades, año 20, nº 39. Primer semestre de 2018. Pp. 39-60. ISSN 1575-6823 e-ISSN 2340-2199 doi: 10.12795/araucaria.2018.i39.03 
Laitin para incluir guerras civiles desde 1816 hasta 1999, Boix concluye que a mayor igualdad en la distribución y mayor grado de industrialización, la probabilidad de ocurrencia de una guerra civil disminuye. "En países en los que las fincas familiares controlan más del $50 \%$ de la tierra cultivada y la industrialización y urbanización ${ }^{9}$ están por encima del $50 \%$, la probabilidad de ocurrencia de una guerra civil en un período de cinco años cae por debajo del 1\%" (Boix 2008, 213). De acuerdo con Stewart (2009), hay un conjunto de características de las desigualdades horizontales que incrementarían el riesgo de conflicto armado:

-A mayor durabilidad, mayor la acumulación de ventajas para unos grupos. Así, el acceso privilegiado de un grupo a mayores ingresos se traduce, por ejemplo, en ventajas educativas para ese grupo.

-Ampliación de las brechas entre los grupos a lo largo del tiempo.

-Las fronteras entre los grupos son lo suficientemente nítidas como para dificultar la movilidad de los individuos entre ellas.

-El grupo en desventaja debe tener un tamaño significativo. Si un grupo en desventaja es demasiado pequeño, puede considerar muy costosa la opción de acudir a la violencia política.

-Si las brechas se expresan consistentemente en varias dimensiones simultáneamente (participación política, activos, oportunidades laborales y de ingresos, acceso a los servicios sociales), los grupos en desventaja estarán más inclinados hacia la rebelión.

-Estancamiento o declive del crecimiento económico de modo que el progreso social y económico de aquellos en situación de privación es nulo en términos absolutos.

-Suficiente cohesión al interior de los grupos para el despliegue de la acción colectiva.

-Si los líderes de grupos en desventaja no son cooptados por el régimen.

-Si los gobiernos no responden a las demandas de los grupos en desventaja o aún peor, si responden a ellas en forma represiva y violenta.

Todas esas características han estado presentes en el caso de los acholi excepto, en cierto grado, la de la consistencia de las brechas entre dimensiones y la de no cooptación de sus líderes por el régimen. Estas excepciones se abordarán con más detalle en la sección 3. Las desventajas sociales y económicas de los distritos del norte de Uganda con respecto al resto del país, son evidentes para cualquiera que viaje por carretera desde Kampala hasta la población de Gulu, capital del distrito del mismo nombre. Al dejar

\footnotetext{
9 Boix usa un índice de diversificación ocupacional como promedio de la mano de obra ocupada en la industria y en el sector urbano.
} 
atrás el central triángulo de Luwero y cruzar el río Nilo que divide al país en dirección sureste-noroeste desde Jinja en el lago Victoria, hasta el lago Alberto, es inevitable no prestar atención al cambio del paisaje social. La pobreza se asoma con mayor intensidad a los bordes de una carretera que se deteriora más a medida que el viajero se interna en tierra acholi. Jimmy Fred Otema, director de la organización Terra Renaissance en Uganda, luego de quejarse del pésimo estado de la infraestructura vial en el norte del país, no titubea para acusar al gobierno: "Museveni no representa un proyecto nacional. Ni siquiera un proyecto regional sino personalista que beneficia a una élite muy reducida de Entebbe"'10.

En 2009 el gobierno de Uganda puso en marcha un plan para la consolidación de la paz en el norte del país al que llamó: Peace Recovery and Development Plan (PRDP). Los objetivos planteados para el PRDP eran fortalecer la presencia del Estado, empoderar a las comunidades, revitalizar la economía y promover la reconciliación. Sin embargo, algunos ven en el PRDP una estrategia a favor del despliegue de procesos de acumulación por desposesión ${ }^{11}$ llevados a cabo por las clientelas del estrecho círculo de Museveni ${ }^{12}$. Al respecto, Francis Arop Omuk, una de las principales voces críticas en Gulu y director ejecutivo de Community Rural Empowerment and Support Organisation, una organización no gubernamental local que trabaja con comunidades rurales en los distritos acholi, afirma:

Después de la guerra, el asunto de la tierra se ha convertido en un serio problema. Muchas vidas se han perdido y muchas tierras han sido confiscadas. El gobierno le ha otorgado miles de hectáreas a Madhvani ${ }^{13}$ para plantaciones de caña de azúcar. Con las privatizaciones que se han llevado a cabo, compañías estatales en los sectores de telecomunicaciones, electricidad y bancario, han sido vendidas a los amigos del gobierno y los costos de estos servicios se han

${ }^{10}$ Entrevista a Jimmy Fred Otema, 23 de septiembre de 2014 en Gulu. Aunque la capital de Uganda es Kampala, la casa oficial y las oficinas del presidente están en Entebbe.

${ }^{11}$ La categoría “acumulación por desposesión” es usada por el geógrafo David Harvey para referirse a una suerte de acumulación originaria permanente que incluye: la mercantilización y privatización de la tierra -relacionada con la expulsión de las poblaciones campesinas; la transformación de varias formas de derechos de propiedad (comunes, colectivos, estatales, etc.) en derechos de propiedad privada exclusivamente; supresión de bienes comunes; supresión de formas nativas y alternativas de producción y consumo; apropiación de activos incluyendo recursos naturales; usura y otras formas de apropiación extorsiva de la fuerza laboral y de los medios de vida de las personas (Harvey 2007).

12 Reno (1999) identifica cómo frente a las reformas de ajuste económico y privatización promovidas por las instituciones financieras internacionales en la década de los noventa, los gobernantes africanos optaron por hipotecar recursos naturales a empresas privadas, sustituyendo parcialmente en unos casos -y caso por completo en otros- la asignación clientelista de empleos y recursos públicos por la adjudicación de tierras y recursos mineros.

${ }^{13}$ En el distrito de Amuru, el grupo Madhvani, una compañía ugandesa vinculada a una familia de origen indio, ha recibido del gobierno un área de cuarenta mil hectáreas para plantaciones de azúcar. En tiempos de Obote, el grupo Madhvani recibió privilegios del gobierno para la administración de empresas nacionalizadas (Kasozi 1994). Ahora, el grupo forma parte del círculo de poder cercano al presidente Museveni. 
elevado bastante. Todo eso ha sido llamado: Programa de Recuperación de la

Paz y el Desarrollo ${ }^{14}$.

UNDP (2015) analiza la situación social y económica en 55 distritos del norte del país cubiertos por el PRDP y evidencia las persistentes disparidades regionales. Esos 55 distritos se dividen en tres categorías: i) severamente afectados por la guerra (que incluye a los acholi); ii) esporádicamente afectados y iii) aquellos cuyos territorios recibieron consecuencias negativas de la guerra, sin haber sido escenario principal o esporádico de ésta. Mientras la expectativa de vida al nacer en el área no cubierta por el PRDP (centro y sur del país) es de 54,6 años, en los distritos severamente afectados es de 53,4 años. El ingreso per cápita en el área no cubierta es de 1525 dólares PPA, y en los distritos severamente afectados es de 707 dólares PPA. El promedio de años de escolaridad en el área no cubierta es 4,9 mientras que en los distritos severamente afectados es 3,3 (UNDP 2015: 180). Esas desventajas, aunque acentuadas por la guerra, la preceden (Kasozi 1994).

Según Lindemann (2011), las desigualdades horizontales relacionadas con los intercambios excluyentes entre las elites (exclusionary elite bargains) que se expresan en las dimensiones política-burocrática, coercitiva, económica y territorial, han estado presentes en el caso de Uganda. En cuanto a la desigualdad entre grupos en el acceso a la burocracia y a los medios de coerción, es necesario advertir que la ventaja inicial a favor de los acholi y su significativa participación en la administración pública a nivel nacional, perdieron vigencia ante la política revanchista puesta en marcha por el NRM desde 1986. Lindemann presenta evidencia tanto del papel desempeñado por las desigualdades horizontales en las guerras de Uganda como de la forma en la que la política de descentralización, basada en la fragmentación de la división política administrativa y la creación de burocracias locales, ha contribuido a disminuir el riesgo de reanudación de guerra civil. Sin embargo, ello no significa la desaparición de la violencia política: "Mientras la política de descentralización del NRM ha ayudado a comprar cierta estabilidad en el corto plazo, esta puede no ser sostenible en el largo plazo" (Lindemann 2011:415).

\section{Identidades étnicas y fracturas sociales en Uganda}

Las desigualdades horizontales que pusieron a los acholi en desventaja tienen raíces muy profundas. Antes de la colonización británica, el sur del país había alcanzado un mayor grado de desarrollo socioeconómico y de centralización política. La mayor densidad poblacional y la necesidad de

\footnotetext{
${ }^{14}$ Entrevista a Francis Arop Omuk, Gulu, 23 de septiembre de 2014.
} 
defender las fértiles áreas y zonas de pastoreo indujeron un mayor grado de cooperación entre los diferentes señoríos del sur. Desde el siglo XV, el poder de hacer la guerra y la paz en el sur fue concentrándose cada vez más en el Kabaka (rey) de Buganda (Kasozi 1994). A fines del siglo XIX, "Buganda era más homogéneo que cualquier otro subestado en la nación, unido por un lenguaje común ${ }^{15}$, costumbres, territorio, estructura de clanes y sobre todo, por la monarquía" (Kasozi 1994: 49).

De otro lado, la identidad acholi fue tomando forma lentamente con la expansión entre distintos señoríos del idioma Luo (de la familia lingüística nilótica). Cada señorío en buena parte del norte de Uganda estaba, desde el siglo XVII, bajo la dirección de un rwot (jefe). Antes de que Uganda pasara a ser protectorado británico en 1894, los acholi estaban bastante conscientes de cierta demarcación identitaria (Atkinson 2010b). Sin embargo, en contraste con Buganda, la naturaleza política de Acholiland era bastante descentralizada:

Los primeros agentes coloniales describieron la tierra acholi como dividida en una docena de señoríos, en cada uno de los cuales, un linaje habría provisto al jefe o rwot-moo (plural: rwodi-moo). Sin embargo, incluso los términos señorío y jefe son ambiguos ya que sugieren una estructura política mucho más centralizada de la que, de hecho, existía (Branch 2011: 47).

El punto es que -aún después de reconocer que la identidad acholi tiene vínculos con un distante pasado y que su proceso de formación tuvo lugar mucho antes del dominio británico- el gobierno colonial jugó un papel decisivo al adjudicar un carácter esencialista a la identidad acholi y a las identidades de otras tribus, "haciendo de la tribu, una categoría política dominante en el escenario político nacional" (Branch 2011: 48).

Para los británicos, Buganda estaba varios peldaños más arriba que los acholi en la escalera de la "civilización", razón por la cual, además de usar el reino más poderoso de Uganda como centro administrativo y comercial para la extensión de su dominio ${ }^{16}$, dieron un tratamiento privilegiado a su élite. Entre tanto, moldearon en forma despótica un proceso de centralización política en Acholiland con el propósito de organizar la extracción de tributos y el reclutamiento de mano de obra. En ese proceso, sustituyeron inicialmente a los rwodi por jefes locales nombrados por el gobierno colonial. Más tarde, con el propósito de apaciguar algunas expresiones de resistencia y cooptar opositores, el gobierno colonial creó los consejos locales y tribales conformados por los rwodi y los jefes nombrados por la administración colonial, así como por representantes de la pequeña burguesía existente entre los acholi (Branch 2011).

${ }^{15}$ El idioma común es el Luganda, perteneciente al complejo lingüístico bantú.

${ }^{16}$ Lo que A.D. Roberts, citado por Atkinson (2010b, 4), llamó “sub-imperialismo Buganda”. 
Además de imponer en forma violenta el modelo de organización política de Buganda en la tierra de los acholi, tanto los británicos como la élite de Buganda, "enfatizaron, exageraron, y algunas veces inventaron distinciones étnicas" (Atkinson 2010b, 4), creando y reforzando estereotipos con los que justificaron una distribución desigual del capital y de la infraestructura. "De hecho, el acceso privilegiado del pueblo Baganda ${ }^{17}$ (especialmente de la élite, a las oportunidades sociales, económicas y políticas, y las muy reales desigualdades materiales que esto generó, crearon aún mayores diferencias y una más fuerte consciencia de las mismas de la que había alguna vez existido antes" (Atkinson 2010b: 4).

El legado de exacerbadas desigualdades regionales que dejó la administración colonial ha contribuido a los altísimos niveles de violencia política experimentados por Uganda desde $1962^{18}$. En la sección anterior se señaló que dos de las características de las desigualdades horizontales que aumentan el riesgo de conflicto armado no han estado del todo presentes en el caso ugandés: la consistencia de las desigualdades horizontales en múltiples dimensiones y la no cooptación de líderes del grupo en desventaja. Uno de los estereotipos sobre los acholi que se forjaron durante el gobierno colonial, estuvo paradójicamente relacionado con un poderoso mecanismo de inclusión política de los mismos: los administradores británicos etiquetaron al pueblo acholi como un pueblo militarista, apto para proveer soldados:

Los británicos notaron también -como muchos otros- que la política colonial favoreció un mayor desarrollo de las regiones del sur del protectorado y descuidó el del norte, lo cual produjo un desequilibrio económico que empujó a los acholi y a otros habitantes del norte a ingresar al ejército (y la policía). Así que, un mítico estereotipo colonial se fusionó con la política colonial para producir un patrón generalizado de existencia. Al momento de la independencia en 1962, el ejército ugandés era predominantemente norteño, con más acholis que miembros de cualquier otro grupo étnico (aunque debe anotarse también que había muchos acholi en puestos profesionales y en la Universidad de Makerere). (Atkinson 2010b: 276).

A pesar de sus desventajas sociales y económicas, había una desigualdad en el control de los medios de coerción que favorecía a los acholi y a otros grupos del norte como el pueblo Langi, al cual pertenecía el presidente Milton Obote. Y la característica de la no cooptación por parte del régimen queda descartada por el hecho de que, entre 1962 y 1971 y entre 1979 y $1986^{19}$,

${ }^{17}$ El pueblo de los Baganda corresponde a los nativos del reino de Buganda.

18 "[E]ntre 1964 y 1985, los ugandeses estuvieron expuestos a un nivel de violencia que supera ampliamente el de cualquier otro pueblo en África oriental. Más de un millón de ugandeses fueron asesinados en este período por razones políticas" (Kasozi 1994: 3).

${ }^{19}$ La dictadura militar de Idi Amin (1971-1979), quien era también del norte pero no era Acholi ni Langi sino que pertenecía al pequeño grupo étnico Kakwa localizado en el noroccidente de Uganda, 
los acholi ocuparon posiciones importantes en los niveles más altos de la administración pública, incluyendo al presidente Tito Okello.

La desigualdad en el acceso a los medios de coerción a favor de los acholi así como su representación, también favorable, en los altos puestos de la administración pública al momento de la independencia, no disminuyeron el riesgo de traducir las desigualdades socioeconómicas, en guerra civil. Al contrario, es posible identificar ambas situaciones como los detonantes de un mecanismo que conectó en el tiempo las desigualdades horizontales con la violencia política: la política despótica de igualación. Mecanismo que se describe más adelante.

Acorde con la perspectiva del institucionalismo histórico, es posible rastrear en el tiempo la forma en la que unas características producen un resultado de interés, de modo que no es simplemente la ausencia o presencia de tales características lo que explica dicho resultado, sino la secuencia específica de eventos y mecanismos que seleccionan, dentro del conjunto posible de resultados (equilibrios múltiples), un equilibrio en particular. De modo que en procesos que se despliegan a lo largo del tiempo, los detalles históricos no son simplemente la ilustración de una expectativa teórica sino la fuente misma de la explicación (Pierson, 2004).

El poder militar y la participación política de los acholi y de los langi en el nivel nacional, detonaron la política de igualación despótica como mecanismo explicativo de la violencia política y de las guerras civiles por una razón: La agresiva política de igualación -viable gracias al acceso privilegiado de los acholi y los langi a los medios de coerción y a la participación política nacionaldibujó un línea divisoria más nítida que las divisiones intrarregionales entre tribus y clanes: una línea entre norte y sur que daría forma a un nivel más agregado de la enemistad política en Uganda. Una distinción que se volvería más tarde contra los acholi y los langi puesto que amplió las opciones de manipulación política de las identidades por parte de los líderes políticos del sur, algo que ha jugado especialmente a favor del presidente Yoweri Museveni.

El primer presidente de la Uganda independiente fue el Kabaka de Buganda, Edward Mutesa II. Milton Obote fue su primer ministro. Pero el poder ejecutivo estaba realmente en las manos de Obote quien, en 1966, usó a Idi Amin para derrocar a Mutesa y suprimió, en 1967, al reino de Buganda y a otros cuatro reinos en el sur. Ese acto puso fin a quinientos años de historia. Incluso antes del golpe contra Mutesa, Obote había estado promoviendo una agresiva política anti-Buganda. Las fuerzas militares - reclutadas principalmente en el

cerca de las fronteras con la RDC y Sudán del Sur, desató una cruenta persecución en contra de los Acholi y los Langi. A pesar de los conflictos entre tribus del norte tanto en el régimen de Amin como en los dos gobiernos de Obote y el breve período de Okello, desde el punto de vista de los bantúes del sur, el ascenso de Museveni al poder en 1986 puso fin a un cuarto de siglo de dominio del norte (Branch 2011). 
norte- fueron instruidas con la idea de que los Baganda -independientemente de su posición social- eran los enemigos de Uganda (Kasozi 1994) ${ }^{20}$.

Desde que los británicos dejaron el poder en manos africanas, Uganda no conoce un cambio pacífico de gobierno. Milton Obote fue derrocado en 1971 por Idi Amin, quien terminó en el exilio en 1979 por cuenta de la invasión llevada a cabo por el gobierno de Tanzania y la guerrilla liderada por Yoweri Museveni. Obote regresó al poder en 1980 y en 1985 fue derrocado por Tito Okello. Museveni siguió combatiendo al gobierno y tras un fallido acuerdo de paz con Okello, entró triunfante a Kampala en 1986, al mando del Ejército Nacional de Resistencia (National Resistance Army NRA). Ha estado en el poder desde entonces; el círculo cercano a Museveni ha contado con la llamada "comunidad internacional" como una fuente adicional de recursos políticos y financieros para el despliegue de sus técnicas de dominación, una característica que -como muestra Reno (1999)- ha sido compartida por gobiernos africanos en el contexto de la posguerra fría. En Uganda esas técnicas y estrategias han estado presentes tanto en la guerra con el LRA como en la fase de "postconflicto" en Acholiland.

El golpe de Amin contra Obote en 1971 -así como el de Okello contra el mismo Obote en 1985- demuestra la persistente importancia de las rivalidades tribales intrarregionales. No obstante, el despótico proyecto de igualación de Obote - menos orientado a promover el desarrollo del norte que a frenar el del sur- terminó introduciendo una cuña entre norte y sur en la política nacional. Una cuña que, a pesar de no proveer una sólida identidad política regional para el norte, brindaría luego - como señala Adam Branch- una base ideológica para la rebelión del NRA de Museveni contra el "Norte".

Como lo explica Kasozi, la política económica del primer gobierno de Obote se orientó hacia la estatización del sesenta por ciento de todas las compañías, industrias e instituciones financieras que operaban en el país, con lo cual, no sólo obstaculizó el surgimiento de una nativa élite empresarial, sino que desmanteló la que ya existía en Buganda. Obote otorgó a empresarios de origen asiático permiso para operar y retener la administración de sus compañías. Estos empresarios contaron no sólo con el apoyo del gobierno en calidad de co-propietario de las mismas para reprimir las demandas de los trabajadores, sino que también tenían un tratamiento privilegiado en el acceso a contratos gubernamentales. Todo ello siempre y cuando la "desafricanización" de la economía ugandesa fuera de la mano con el desmantelamiento de la economía de Buganda (Kasozi 1994: 90-92).

${ }^{20}$ A.B. K. Kasozi destaca un evento ilustrativo de los alcances de esta política de adoctrinamiento contra los Baganda, el cual ocurrió en mayo de 1964 en el vecindario de Nakulabye en Kampala. La policía acudió al llamado de los vecinos por un caso de violencia doméstica. Sin hacer mayores averiguaciones, la policía disparó contra los vecinos. Los uniformados gritaban a la multitud: "Ustedes los Baganda son unos engreídos. Ustedes no nos traen sino problemas". Seis personas fueron asesinadas esa noche por las fuerza de seguridad y ninguno de los oficiales fue enjuiciado por asesinato o abuso de poder (Kasozi 1994: 77-79). 
La política de Obote no le permitió ganar el apoyo del campesinado (ni del norte ni del sur) puesto que no reformó la estructura despótica de los consejos locales heredada de la colonia (Branch 2011). Obote alentó además la agricultura de gran escala en manos de monopolios estatales y compañías como el grupo Madhavani (Kasozi 1994). Como explica Branch, el gobierno de Obote se dedicó a comprar apoyo mediante una política patrimonialista ejecutada por su partido político, el Congreso del Pueblo Africano (Uganda People's Congress UPC). Política de la que se beneficiaban algunos rwodi, parte de la pequeña burguesía acholi y langi, y algunos de los jefes nombrados desde la administración colonial.

Luego, el errático régimen de Idi Amin contribuyó a exacerbar una de las características de las desigualdades horizontales que aumentan el riesgo de guerra civil: El declive del crecimiento económico y el aumento generalizado de los niveles absolutos de privación de la población en general. Tras la caída de Amin, Obote regresa al poder en medio de acusaciones de fraude electoral ante lo cual, se desata la guerra civil entre el NRA liderado por Museveni y las fuerzas gubernamentales del Ejército Nacional de Liberación de Uganda (Uganda National Liberation Army UNLA).

Como explica Atkinson (2010b), el UNLA llevó a cabo una brutal política contrainsurgente en la zona central de Uganda que corresponde al llamado triángulo de Luwero, aumentando el resentimiento antigubernamental, hábilmente capitalizado por el NRA. Los combatientes del NRA adoptaron la práctica de referirse a los soldados del UNLA y al gobierno de Obote como los "acholis", práctica adoptada igualmente por la población de Luwero en general. Así, identificando y etiquetando un enemigo común, el NRA fue promoviendo, por contraste, la conciencia de una común identidad bantú entre la heterogénea población de Luwero que incluía refugiados tutsis provenientes de Ruanda ${ }^{21}$, gentes del pueblo Baganda (antiguos súbditos del Kabaka Mutesa) y miembros del pueblo Banyankole (del reino de Ankole) -al que pertenece Museveniprovenientes de Uganda occidental (quienes también tenían representación en las filas del UNLA). Museveni hábilmente disminuyó el rol de esas identidades promoviendo la creación de Consejos de Resistencia multiétnicos, lo que le permitió enfatizar cada vez más la enemistad política en términos de la dicotomía bantúes versus nilóticos (acholis).

Luego de la disputa "intranilótica" entre acholis y langis que llevó al derrocamiento de Obote y al efímero gobierno de Okello, las fuerzas de Museveni tomaron el poder en Kampala y persiguieron al UNLA hasta la frontera norte del país. La llegada masiva de los soldados del derrotado UNLA a la tierra acholi resultó demasiado disruptiva. Los ancianos líderes tradicionales de los acholi consideraban a estos jóvenes como una amenaza.

${ }^{21}$ De hecho, Paul Kagame, el actual presidente de Ruanda, estuvo en las filas del NRA. 
Esas autoridades tradicionales, "como explica la antropóloga Heike Behrend, veían a quienes habían retornado como la causa de todo mal. Eran extraños a los ojos de quienes habían permanecido en casa. Ellos habían saqueado, asesinado y torturado en Luwero. Sus corazones eran ahora impuros" (Branch 2010: 32). Sin embargo, buena parte de los excombatientes se rehusó a aceptar la autoridad y los rituales de limpieza exigidos por los líderes tradicionales. La etnia acholi ahora estaba escindida, sus desventajas sociales y económicas persistían, y su acceso privilegiado a los medios de coerción y a la política nacional, se había perdido.

\section{Contrainsurgencia y destrucción en Acholiland}

El NRA convertido en el ejército del gobierno pasó a ser el UPDF y como partido de Museveni pasó a ser el Movimiento Nacional de Resistencia (National Resistance Movement NRM). El nuevo gobierno no tomó nota de los conflictos al interior de los acholi y de las dificultades de los excombatientes del UNLA para reintegrarse a la sociedad. En cambio, consideraba que la población de Gulu, Kitgum, Pader y Amuru, apoyaría decididamente al UNLA en un temprano proceso de reorganización y levantamiento armado contra el gobierno. Así que el NRA (luego UPDF) llevó a cabo una política de tierra arrasada en los distritos acholi, dando lugar a lo que Adam Branch identifica como una agresiva estrategia de contrainsurgencia sin insurgencia. Ese fue el inicio de la nueva guerra civil en Uganda: "La guerra comenzó por varios factores, entre ellos, la masiva violación de derechos humanos por parte del gobierno actual. Cuando ellos llegaron al poder buscaban venganza"22. Las palabras de Otema son ratificadas por Francis Arop Omuk:

El NRA vino con una mentalidad de venganza. Su estrategia consistió en acosar y ultrajar a las personas de la comunidad, fueran excombatientes o no. Esto llevó a la gente a pensar que ellos habían llegado para exterminar a los acholi. En respuesta, la comunidad empezó a unirse libremente a los focos de rebeldes, buscando protección. Teníamos varias facciones rebeldes. Una de ellas, llamada Ejército Democrático del Pueblo de Uganda (Uganda People Democratic Army UPDA) era un producto del UNLA. Luego surgieron el Movimiento del Espíritu Santo (Holy Spirit Movement HSM) y el LRA ${ }^{23}$.

Una vez resultó claro que el UPDA no podía proveer suficiente protección ni frente al NRA ni frente al robo de ganado llevado a cabo por los Karamojong, quienes despojaban a los acholi de sus medios de vida con la total aquiescencia

${ }^{22}$ Entrevista a Jimmy Fred Otema, 23 de septiembre de 2014 en Gulu.

${ }^{23}$ Entrevista a Francis Arop Omuk, Gulu, 23 de septiembre de 2014. 
del NRA, el UPDA perdió el respaldo de la población. Su lugar fue tomado por el HSM liderado por una médium: Alice Auma conocida como Alice "Lakwena" (mensajera) ${ }^{24}$. El HSM alcanzó a reunir entre siete mil y diez mil soldados y avanzó hacia Kampala, logrando una temporal alianza anti-Bantú, la cual, una vez en el sur, enfrentó la hostilidad de la población y fue derrotada por el NRA. En un contexto de disputas violentas entre remanentes del UPDA y del HSM, surge el LRA:

Cuando Lakwena abandonó Acholiland, la violencia entre diferentes facciones rebeldes se intensificó. Aunque las fuerzas de Lakwena generalmente evitaban la violencia contra los civiles, fragmentos del UPDF y algunas derivaciones del HSM, aterrorizaban a sus supuestas bases sociales entre sí. Adicionalmente, una vez Lakwena había agotado la afluencia de voluntarios, esas facciones tenían que aumentar el reclutamiento forzado. Es ese el ambiente en el que Kony emergió (Branch 2011: 68).

Joseph Kony dependía fundamentalmente del reclutamiento forzado. Así que ejerció cada vez más violencia contra los civiles de los distritos de Gulu, Kitgum, Pader y Amuru. De acuerdo con Fred Bongomin, empleado de un restaurante y estudiante de la Universidad de Gulu: "Al comienzo, el LRA tenía un carácter protector. Después, empezó a atacar a su propia gente. La comunidad perdió la confianza en el LRA y en los grupos que le precedieron porque nunca lograron proteger al pueblo acholi" 25 .

Según Branch, el gobierno de Museveni dejó que los rebeldes atacaran a la población civil, en parte para castigar a los acholi y en parte como una estrategia para evitar que los rebeldes construyeran una base social. Cuando los grupos restantes del UPDA firmaron, en junio de 1988, un acuerdo de paz con el gobierno, Kony estableció una nueva distinción entre "verdaderos" y "falsos" acholis a partir de la cual, el LRA exacerbó la violencia contra los civiles en el norte de Uganda.

Como se mencionó en la introducción, el apoyo del régimen de Jartum le permitió al LRA sostener su desafío al gobierno de Museveni en el norte de Uganda por casi veinte años. "La dependencia del LRA de fuentes externas le evitó la necesidad de cultivar apoyo local" (Reno 1999: 69). Luego de los fallidos diálogos de paz en 1994, el LRA escaló sus ataques. El gobierno ugandés proclamaba, al inicio de cada contraofensiva, que el final de Kony y sus hombres estaba cerca. Sin embargo, una y otra vez el LRA regresaba de Sudán e intensificaba sus acciones. Museveni resolvió poner en marcha en 1996 una nueva y devastadora política contrainsurgente que hizo trizas, no al LRA, sino a los modos y medios de vida de los acholi.

24 "Ella difundió un discurso de limpieza espiritual en Acholiland, basándose en una larga tradición alternativa a la que reivindicaban los viejos jefes, lo que le permitió afirmar su autoridad sobre el UPDA, los ex-UNLA y sobre los civiles acholi en general” (Branch 2010, 36).

25 Entrevista a Fred Bongomin, Gulu, 24 de septiembre de 2014. 
En septiembre de ese año, so pretexto de proteger a los civiles de la violencia ejercida por el LRA, el UPDF desplazó forzosamente a los acholi de sus tierras para internarlos en "villas protegidas". En realidad, se trataba de campos de desplazados en los que los acholi fueron sometidos no sólo a condiciones sanitarias deplorables, sino también a los abusos y a la violencia ejercida contra ellos por las fuerzas gubernamentales: "La población total de esos campos se situaba en unos cientos de miles al finalizar 1996, pero para mediados de la década de los 2000 alcanzaba aproximadamente un millón, abarcando casi toda la población rural de la subregión acholi" (Branch 2011: 92) ${ }^{26}$.

Museveni solicitó el apoyo del Programa Mundial de Alimentos y otras agencias humanitarias. Adam Branch sostiene que dicho apoyo tuvo no sólo el efecto indeseado de contribuir a la prolongación de la guerra, sino de hacer viable la estrategia contrainsurgente del gobierno. Esos campos de desplazados se mantuvieron debido a la violencia del estado ugandés que llevó a la fuerza a la gente a esos lugares y a "la intervención de las agencias humanitarias, que los administraron y sostuvieron por casi una década” (Branch 2011: 91).

Tomando nota del difícil dilema moral enfrentado por las agencias humanitarias, el profesor Phillip Apuuli Kasaija de la Universidad de Makerere afirma: "La ayuda prolongó las vidas de la gente en los campos con lo cual también contribuyó a la prolongación de la guerra. Pero usted no puede culpar a las organizaciones humanitarias por brindar asistencia a la gente en los campos"27. No obstante, Branch cuestiona ese dilema moral con un argumento contrafáctico: si las agencias no hubieran acudido al llamado de Museveni, éste habría tenido que desmantelar los campos al poco tiempo. En efecto, la gente habría preferido mantener su principal medio de vida, la tierra, aún con la incertidumbre de ser atacada por el LRA ${ }^{28}$, ante la certidumbre de quedar sometido a la violencia ejercida por las fuerzas gubernamentales y perderlo todo.

Aunque los campos fueron desmantelados en 2006, sus efectos moldean los conflictos y la violencia social que permea el "postconflicto" en el norte de Uganda. De acuerdo con Ojara Martin Mapenduzi, Presidente del Distrito de Gulu, una de las consecuencias más dramáticas de los campos ha sido la pérdida de la capacidad que solían tener, los ancianos líderes tradicionales, para arbitrar los conflictos cotidianos de la gente:

${ }^{26}$ El 29 de agosto de 2005, Jan Egeland, Subsecretario General de Naciones Unidas para Asuntos Humanitarios calificó la situación en el norte de Uganda como la peor crisis humanitaria en el mundo.

27 Entrevista realizada en Kampala, el 30 de septiembre de 2014.

28 De hecho, estar en los campos no los libraba de los ataques del LRA. De acuerdo con Francis Arop Omuk, en cierto modo el eufemístico nombre de "villas protegidas" tenía sentido: Las comunidades eran ubicadas de modo que circundaban los campos de desplazados, mientras el UPDF permanecía al interior. El que estaba protegido era el ejército: "La gente en realidad protegía al ejército, en lugar de estar protegida por este. La comunidad permanecía atrapada en medio del fuego cruzado". Entrevista realizada en la población de Gulu el 23 de septiembre de 2014. 
Tradicionalmente, los viejos eran una fuente de autoridad. Se supone que estaban para proveer un sentido de dirección a la comunidad, mediar en los conflictos y contribuir a la reconciliación. Pero ellos ya no desempeñan ese rol tradicional. Las cosas han cambiado. En el pasado, cuando un hombre quería casarse y ni él ni su padre tenían cómo pagar la dote, el abuelo ayudaba. Las autoridades tradicionales dependían de su ganado y ahorraban manteniendo sus animales y eso, les daba poder... Ahora, la gente no les presta atención porque los viejos se han vuelto dependientes. Perdieron sus animales y se convirtieron en mendigos. De manera que ese respeto, esa autoridad, esa clase de dirección que ellos solían proveer ya no es visible hoy ${ }^{29}$.

Los efectos positivos ${ }^{30}$ que tendría el debilitamiento de la autoridad tradicional, especialmente para las mujeres y los jóvenes, se ven opacados por la creciente conflictividad social en las zonas rurales y en centros urbanos que, como la población de Gulu, padecen las consecuencias disruptivas asociadas al declive generalizado del acceso a medios de vida. Como se explica en la siguiente sección, los conflictos de tierras, los enormes obstáculos que enfrentan quienes estuvieron en el LRA o literalmente "nacieron" en sus campamentos, y la severa falta de oportunidades laborales en la tierra acholi, son características que moldean una situación de paz violenta en el norte de Uganda, en un contexto nacional de acentuado autoritarismo político.

\section{Paz represiva y violenta}

Yoweri Museveni, al igual que otros líderes africanos como el presidente ruandés Paul Kagame ${ }^{31}$, ha logrado posicionarse bien ante los ojos de occidente $\mathrm{y}$ de las instituciones financieras internacionales mientras consolida, con la aquiescencia de la comunidad internacional, la estabilidad en el ejercicio de su autoritario poder político. Uganda se mantuvo como un régimen "sin partidos políticos"32 desde 1986 hasta 2005. Los partidarios del NRM, en la práctica el

\footnotetext{
${ }^{29}$ Entrevista realizada a Ojara Martin Mapenduzi, Presidente del Distrito de Gulu en la sede del gobierno distrital el 25 de septiembre de 2014.

30 "Para algunos, especialmente jóvenes y mujeres, la vida en la ciudad (Gulu), a pesar de las privaciones materiales, es vista como la base de un mundo global y moderno, opuesto a un mundo local y tradicional, que ofrece cierto grado de libertad, independencia y oportunidad que era imposible en las villas"

${ }^{31}$ Para el caso de la transición del genocidio a la paz represiva en Ruanda ver: Samset (2011).

${ }^{32}$ La política de "democracia de movimiento" puesta en marcha hasta su rechazo en el referéndum constitucional de 2005, implicaba que las personas podían competir a título individual en las elecciones para conformar consejos locales y regionales, dentro de los límites impuestos por el NRM. Al principio se trataba de una estrategia que prometía empoderar a la gente común del campo y las pequeñas localidades en la transición del NRM de guerrilla a gobierno civil. Sin embargo, en el año 2000 un analista hacía la siguiente observación: "Durante las crisis políticas, los líderes del NRM con frecuencia usan su control sobre la 'democracia de movimiento' como un arma para afianzar su propio poder... La única constante a lo largo de los años en la 'democracia de movimiento' ha sido la
} 
partido de gobierno, señalaban que la competencia multipartidista pondría en riesgo la unidad nacional y la reconciliación tras la guerra civil de 1981-1985 (Mugaju y Oloka-Onyango 2000). Ciertamente, como lo ha mostrado Paris (2004), la rápida liberalización política tras la terminación de una guerra civil, aumenta el riesgo de recaída en la guerra o de reanudación de la violencia social o política. De modo que es necesario poner en funcionamiento las instituciones apropiadas para la adecuada regulación de la competencia electoral ${ }^{33}$. Sin embargo, claramente un régimen sin partidos políticos por diecinueve años y sin alternancia en el poder por treinta años, corresponde más a un caso de crudo autoritarismo político que a una estrategia de institucionalización previa a la liberalización.

A pesar de la proscripción de la actividad de los partidos políticos, el índice de Freedom House calificó a Uganda en forma ininterrumpida, desde 1994, como un país "parcialmente libre". Sin embargo en 2015, recibió la calificación de "no libre". De hecho, las elecciones del 18 de febrero de 2016 en las que Museveni obtuvo su quinto período como presidente del país, con $61 \%$ de la votación (su principal opositor y antiguo aliado Kizza Besigye, del Foro por el Cambio Democrático recibió 35,6\%) han sido fuertemente cuestionadas por denuncias de fraude, violencia y actos de intimidación contra los candidatos de la oposición, entre ellos, el arresto domiciliario del propio Besigye (Butagira 2016). "En cada elección -afirma el profesor Phillip Apuuli Kasaija- el gobierno ha usado mucho la violencia. En las elecciones de 2011 la violencia no fue tan intensa porque el gobierno usó mucho dinero"34.

Entre tanto, a pesar de que el LRA no es una amenaza visible, la situación en el norte de Uganda difícilmente puede calificarse como pacífica. James Okello, directivo de Human Rights Focus en Gulu afirma: "No diría que Gulu está realmente en paz. Hay violencia relacionada con un sinnúmero de conflictos por la tierra. Muchos rwodi que conocían los linderos de las tierras de cada clan murieron en los campos de desplazados. Así que quienes regresaron no saben exactamente cuál es su tierra"35. Otra fuente de conflicto según James Okello, está relacionada con el acceso selectivo a los programas sociales del gobierno, los cuales parecen más orientados por la búsqueda de un mínimo apoyo político local que por criterios técnicos de focalización. En todo caso, cualquier estrategia de focalización en los distritos acholi, sería equivalente a tratar de iluminar un cuarto totalmente oscuro con una pequeña linterna:

prohibición de la actividad política de los partidos. Con el tiempo, este rechazo a la actividad partidista se ha tornado cada vez más vacío y más relacionado con consideraciones de poder que con razones democráticas" (Kasfir 2000:61).

${ }_{33}$ Lo mismo aplica, en el análisis de Paris, a la cuestión de la liberalización económica.

34 Entrevista en Kampala, 30 de septiembre de 2014.

35 Entrevista en la sede de Human Rights Focus en Gulu, 25 de septiembre de 2014. 
La tasa de desempleo en el distrito de Gulu está entre 65 y 70 por ciento. Hay un sinnúmero de factores asociados, entre ellos el hecho de la que la tasa de dependencia es muy alta: de cada diez personas, siete necesitan ayuda para acceder a atención médica, a la educación o simplemente para comer. Ese es uno de los legados de los campos de desplazados porque a la gente no se le permitía ir a cultivar ni a buscar sus propios medios de vida. Dependían totalmente de la ayuda humanitaria. Los campos hicieron improductiva a la gente ${ }^{36}$.

Sin embargo, no se trata simplemente de una suerte de cultura de la dependencia creada por los campos de desplazados, sino de las severas restricciones que tienen los acholi para acceder a medios de vida, especialmente a la tierra. Los conflictos de tierras no están relacionados sólo con las disputas entre clanes o el rechazo del que son víctimas en las villas, los jóvenes y las mujeres que regresaron tras haber sido raptados por el LRA (además de aquellos que nacieron en sus campamentos). También tienen que ver con la estrategia de acaparamiento puesta en marcha por el gobierno para favorecer los intereses de quienes, como el grupo Madhvani, pertenecen al círculo de amigos de Museveni ${ }^{37}$.

Las operaciones de acaparamiento de tierras en Uganda incluyen a gobiernos extranjeros, corporaciones de alimentos y biocombustibles, emporios azucareros como Madhvani, inversiones para el aseguramiento de reservas de caza con fines turísticos, etc. Esas adquisiciones de tierra suelen representar grandes beneficios para funcionarios gubernamentales y militares de alto rango y para empresarios cercanos al gobierno (Martiniello 2013). De acuerdo con Martiniello, la ideología en la que se basan estas operaciones de acaparamiento presenta la tierra de los acholi en particular, y a África en general, como enormes espacios de tierra no utilizada y abundante. Pero esos no son espacios vacíos, son tierras en las que viven (y de las que viven) los acholi, y de las cuales salen por la amenaza del uso de la fuerza, por la brumosa evidencia de la posesión resultado de diez años de confinamiento en los campos de desplazados, y por la carencia absoluta de medios para acceder a servicios médicos. Esto último los lleva a vender la tierra a cambio de algo de dinero en circunstancias de extrema necesidad, de las que los compradores fácilmente sacan partido.

\footnotetext{
${ }^{36}$ Entrevista realizada a Ojara Martin Mapenduzi, Presidente del Distrito de Gulu en la sede del gobierno distrital, el 25 de septiembre de 2014.

${ }^{37}$ Una buena identificación de algunos de los miembros más prominentes de ese círculo está en la nota periodística de Moses (2014). Allí aparece la familia Madhvani y su líder Manubhai Madhvani. También están la familia Mulwana, Patrick Bitature, uno de los hombres más ricos del país, la familia Alam, entre otros.
} 


\section{Conclusión}

Las guerras en el norte de Uganda ilustran bastante bien el vínculo entre desigualdades horizontales y violencia política. Se trata de un vínculo que no es automático. En cambio, es activado por decisiones políticas que actúan como mecanismos explicativos específicos. En el caso de Uganda, esas decisiones políticas han sido básicamente dos: Primero, la agresiva política anti-Buganda llevada a cabo por los gobiernos de Obote-en un contexto de intensas rivalidades tribales inter e intrarregionales- y su despótico proyecto de igualación por lo bajo entre el norte y el sur. Segundo, la despiadada política contrainsurgente puesta en marcha por Yoweri Museveni en el norte de Uganda. Política cuya viabilidad y sostenibilidad dependió, paradójicamente, de la intervención de las agencias humanitarias.

Las desigualdades horizontales en Uganda han sido persistentes en las dimensiones social y económica. No obstante, en las dimensiones de acceso a la burocracia y a los medios de coerción, han sido cambiantes. Esto se relaciona con las decisiones políticas que reforzaron la distinción y la enemistad entre nilóticos y bantúes. En efecto, la manipulación política de las identidades regionales y lingüísticas, conectó las desigualdades horizontales con las guerras civiles que tuvieron lugar en Uganda entre 1981 y 1985 y entre 1985 y 2006.

El postconflicto en el norte de Uganda está plagado de violencia. Se trata no sólo del legado violento de dos décadas de guerra civil, sino también, de los conflictos sociales asociados a la persistencia de las desigualdades horizontales y a los conflictos de tierras exacerbados por la política de acaparamiento promovido por el gobierno nacional. Tanto por las abundantes manifestaciones de violencia directa, como por la violencia estructural ejercida contra los acholi, el norte de Uganda es hoy un ejemplo conspicuo de paz violenta, represiva e inestable. Como bien advierte Lindemann (2011:416): "la guerra civil podría ser remplazada por diferentes formas de conflicto violento". 


\section{Referencias bibliográficas:}

Allen, Tim y Koen Vlasenroot. (2010). Introduction. En The Lord's Resistance Army. Myth and reality, eds. Tim Allen y Koen Vlasenroot, 1-21. New York: Zed Books.

Atkinson, Ronald. (2010a). 'The realists in Juba'? An analysis of the Juba peace talks. En The Lord's Resistance Army. Myth and reality, eds. Tim Allen y Koen Vlasenroot, 205-222. New York: Zed Books.

Atkinson, Ronald. (2010b). The roots of ethnicity. Origins of Acholi of Uganda. Kampala: Fountain Publishers.

Boix, Carles. (2008). Civil wars and guerrilla warfare in the contemporary world: toward a joint theory of motivations and opportunities. En Order, conflict and violence, eds. Stathis Kalyvas, Ian Shapiro y Tarek Masoud, 197-218. Cambridge: Cambridge University Press.

Branch, Adam. (2010). Exploring the roots of LRA violence: political crisis and ethnic politics in Acholiland. En The Lord's Resistance Army. Myth and reality, eds. Tim Allen y Koen Vlasenroot, 25-44. New York: Zed Books.

Branch, Adam. (2011). Displacing human rights. War and intervention in northern Uganda. Oxford: Oxford University Press.

Brubacher, Matthew. (2010). The ICC investigation of the Lord's Resistance Army: An insider's view. En The Lord's Resistance Army. Myth and reality, eds. Tim Allen y Koen Vlasenroot, 262-277. New York: Zed Books.

Butagira, Tabu. (2016). Ugandans are desperate for democracy, Yoweri Museveni only gives them tyranny. The Guardian, 22 de febrero. http:// www.theguardian.com/commentisfree/2016/feb/22/uganda-democracyyoweri-museveni-tyranny-president-force-intimidation

Cederman, Lars-Erik, Kristian S. Gleditsch y Halvard Buhaug (2013). Inequality, Grievances and Civil War. New York: Cambridge University Press.

Frankema, Ewout. (2009). The distribution of land holdings by country. En Poverty, inequality and policy in Latin America, eds. Stephan Klasen y Felicitas Nowak-Lehman, 303-309. Cambridge: The MIT Press.

Harvey, David. (2007). Neoliberalism as creative destruction. Annals of the American Academy of Political and Social Science 610: 22-44.

Kanbur, Ravi y L. Squire. (2001). La evolución del pensamiento sobre la pobreza. Explorando las interacciones. En Fronteras de la economía del desarrollo. El Futuro en perspectiva, eds. Gerald Meier y Joseph Stiglitz, 173-217. Bogotá: Banco Mundial. 
Kasfir, Nelson. (2000). 'Movement' democracy, legitimacy and power in Uganda. En No-party democracy in Uganda. Myths and realities, eds. Justus Mugaju y J. Oloka-Onyango, 60-78. Kampala: Fountain Publishers.

Kasozi, A.B.K. (1994). The social origins of violence in Uganda. Kampala: Fountain Publishers.

Lindemann, Stefan (2011). Just another change of guard? Broad-based politics and civil war in Museveni's Uganda. African Affairs, 110 (440): 387-416.

Martiniello, Giuliano. (2013). Accumulation by dispossession. Agrarian change and resistance in northern Uganda. Working paper No. 12. Makerere Institute of Social Research. Makerere University.

Moses, Mubiru. (2014). 10 Ugandan family businesses and what we learn from them. The Business Owner Magazine, 2(17), 14-18.

Mugaju, Justus y J. Oloka-Onyango. (2000). Introduction: revisiting the multiparty versus movement system debate. En No-party democracy in Uganda. Myths and realities, eds. Justus Mugaju y J. Oloka-Onyango, 1-7. Kampala: Fountain Publishers.

Samset, Ingrid. (2011). Building a repressive peace: The case of post-genocide Rwanda. Journal of Intervention and Statebuilding 5 (3): 265-283

Stewart, Frances. (2009). Policies towards horizontal inequalities in postconflict reconstruction. En Making peace work: the challenges of social and economic reconstruction, eds. Tony Addison y Tilman Brück, 146174. New York: Palgrave-Macmillan.

Paris, Roland. (2004). At war's end. Building peace after civil conflict. Cambridge: Cambridge University Press.

Pierson, Paul. (2004). Politics in time. History, institutions and social analysis. Princeton: Princeton University Press.

Reno, William (1999). Warlord politics and African states. London: Lynne Rienner Publishers.

UNDP. (2015). Uganda human development report. Unlocking the development potential of northern Uganda. Kampala: UNDP.

Uribe López, Mauricio. (2013). Civil wars and violent peace in Africa and Latin America: a general outlook. Africa Peace and Conflict Journal 6(3): 50-63. 